Review

\title{
Effects of caloric restriction on the antagonistic and integrative hallmarks of aging
}

\author{
Begun Erbaba $^{\mathrm{a}, \mathrm{b}}$, Ayca Arslan-Ergul ${ }^{\mathrm{b}}$, Michelle M. Adams ${ }^{\mathrm{a}, \mathrm{b}, \mathrm{c}, *}$ \\ ${ }^{a}$ Interdisciplinary Graduate Program in Neuroscience, Aysel Sabuncu Brain Research Center, Bilkent University, Ankara, Turkey \\ ${ }^{\mathrm{b}}$ National Nanotechnology Research Center (UNAM), Bilkent University, Ankara, Turkey \\ ${ }^{\mathrm{c}}$ Department of Psychology, Bilkent University, Ankara, Turkey
}

\section{A R T I C L E I N F O}

\section{Keywords:}

Brain

Aging

Calorie restriction

Neuroinflammation

Cell cycle

\begin{abstract}
A B S T R A C T
Aging is a significant risk factor for cognitive decline associated with neurodegenerative diseases, which makes understanding what promotes 'healthy brain aging' very important. Studies suggest that caloric restriction (CR) is a non-genetic intervention that reliably extends life- and healthspan. Here, we review the CR literature related to both the subject of aging and alterations in cell cycle machinery, especially surrounding the regulation of the E2F/DP1 complex, to elucidate the cellular protection mechanisms in the brain induced via dietary applications. The alterations extending lifespan via CR appear to exert their effects by promoting survival of individual cells, downregulating cell proliferation, and inducing stem cell quiescence, which results in keeping the stem cell reserve for extreme needs. This survival instinct of cells is believed to cause some molecular adaptations for their maintenance of the system. Avoiding energy waste of proliferation machinery promotes the long term survival of the individual cells and this is due to adaptations to the limited nutrient supply in the environment. Such a protective mechanism induced by diet could be promoted via the downregulation of crucial cell cycle-related transcription activators. This review article aims to bring attention to the importance of molecular adaptations induced by diet that promote healthy brain aging. It will provide insights into alternative targets for new treatments or neuroprotective approaches against neurodegenerative pathophysiologies.
\end{abstract}

\section{Introduction}

Aging is an inevitable biological process and a very complex state. Even in the healthy individual, it can induce numerous alterations such as cellular senescence, telomere attrition, stem cell exhaustion, loss of protein homeostasis; as well as creating complexities such as genomic instability, epigenetic alterations, deregulated nutrient sensing, altered intercellular communication, and mitochondrial dysfunction (Jones et al., 2014; López-Otín et al., 2013). Gene expression levels also have been shown to become more variable during aging, not at the level of individual genes, but being widespread throughout the transcriptome level (Somel et al., 2006). Both genetic and environmental factors contribute to brain aging, which is why scientific research keeps searching for the most effective and easy to apply approaches to counteract the aging process. Numerous studies have shown that caloric restriction has an effect on several cellular features related to normal aging (Fig. 1). In this review we discuss the relationship of caloric restriction to deregulated nutrient sensing, cellular senescence, stem cell exhaustion, and altered intercellular communication. We will start by defining different dietary interventions, and then summarize the findings observed following aging and caloric restriction, and lastly, we will focus on a transcription factor, DP1, which we consider worthy of further investigation.

\section{Dietary regimens}

A great deal of work has focused on dietary interventions, which in this review, we will classify into three types: 1) caloric restriction (CR), 2) intermittent fasting (IF), and 3) ketogenic diet (KD). While caloric restriction is being classified into its own category, it is related to both IF and $\mathrm{KD}$. In the IF dietary regimen, the fasting period usually results in a total reduction of daily caloric intake and in the $\mathrm{KD}$, since carbohydrates are not included and protein based food has less calories, this also results in a caloric reduction. However it is clear from the current research that both IF and KD dietary interventions have their own exclusive health benefits.

\footnotetext{
* Corresponding author at: Interdisciplinary Graduate Program in Neuroscience, Aysel Sabuncu Brain Research Center, Bilkent University, Ankara, Turkey.

E-mail address: michelle@bilkent.edu.tr (M.M. Adams).
} 


\subsection{Caloric restriction}

Caloric restriction (CR) is typically a daily decreased nutrient uptake intervention that is reached by applying a diet with a $30-40 \%$ reduction in overall caloric intake without causing malnutrition for a particular duration. Effects of CR on increased life- and healthspan, as well as cellular senescence, have been studied in diverse species such as budding yeast, flies, spiders, worms, fish, and monkeys (Al-Regaiey, 2016; Arslan-Ergul et al., 2013; Pani, 2015; Weithoff, 2007). It was reported that CR helps not only to slow down the aging process but also increases cognitive performance, protects against the deterioration of tissue structure and function, as well as maintaining stem cell function that otherwise declines sharply with age. Moreover, it reduces DNA damage and promotes an anticancer response along with applied chemotherapeutic drugs during cancer treatment (Cerletti et al., 2012; Meynet and Ricci, 2014). The effects of CR also have been shown to present differential effects on different tissues (Arslan-Ergul et al., 2016). Such cell protective responses induced via CR present in nearly all organs, including the brain.

The overall effectiveness of CR seems to be modulated by several factors, including dietary composition, the duration of the applied dietary regimen, and the time of initiation of the diet. In terms of the dietary composition, specific lipid profiles and signals have been suggested to delay aging (de Diego et al., 2019), and these emphasize the role of dietary composition and metabolism in affecting aging in the brain. Several scientific reports on the outcomes related to the initiation and duration of CR have also been tested, and the conclusion is that CR applied in the first or second half of life was more effective than application during both halves (Feldman, 2013). A study using rotifer species demonstrated that increases in the length of CR to more than the median age of the animal extends the lifespan by a concomitant decrease in the lifetime reproduction (Weithoff, 2007). Therefore, CR has been proposed to be a beneficial approach that should be investigated further to improve healthy brain aging.

\subsection{Intermittent fasting}

Intermittent fasting (IF) is a regimen in which there are repeating cycles of ad libitum eating and fasting. The fasting period might be as long as $20 \mathrm{~h}$. There are various types of IF with durations of $12 \mathrm{~h}$ eating and $12 \mathrm{~h}$ fasting, to $4 \mathrm{~h}$ eating and $20 \mathrm{~h}$ fasting or it can be applied weekly with 5 days eating and 2 days fasting. During the periods of fasting, cells activate the pathways that otherwise may remain latent such as glucose regulation, stress resistance, and control of reactive oxygen species (ROS), as well as the downregulation of inflammation (de Cabo and Mattson, 2019). IF resembles the feeding regimen of prehistoric humans since they did not have constant access to food and had to endure long fasting periods. This is in contrast to modern civilization where people have constant access to food, which can be a risk factor for aging-related disorders, including neurodegenerative disorders (Mattson et al., 2017). For example, IF has the potential to reduce cognitive defects and improve memory performance by increasing brain plasticity through the regulation of synaptic proteins and the increase in the production of brain derived neurotrophic factor (BDNF), the major mediator of neuroplasticity and neuronal maintenance (Mattson, 2005; Cherif et al., 2016; Liu et al., 2019). After a period of an IF regimen, the body adapts to evolutionary-conserved metabolism that is mediated through GABAergic synaptic activity (Liu et al., 2019). Another application of IF is time-controlled fasting (TCF), which have extended periods of fasting followed by periods of normal diet. This has been tested in mice, and as opposed to a ketogenic diet, which is discussed below, TCF reduces glucose and insulin levels and increases mitochondrial flexibility through the regulation of the rate-limiting enzyme of cellular triglyceride hydrolysis (Lettieri-Barbato et al., 2018). Taken together IF has the possibility to have multiple beneficial effects on deterring brain aging, and this type of dietary regimen may be easier for translation to human populations.

\subsection{Ketogenic diets}

After the application of a ketogenic diet (KD), a high-fat, low-carbohydrate regimen, the body switches into starvation mode. In this situation the body uses fat as the energy source, and produces ketones. A KD has long been a treatment option for drug resistant epilepsy, and been prescribed to children and adults with this disorder (Lyons et al., 2020). KDs were suggested to be neuroprotective on the aging brain cells by reducing inflammation (Rusek et al., 2019). For this reason, recently ketone-inducing therapies have been suggested as a treatment options to alleviate some of the Alzheimer's disease symptoms (Taylor et al., 2019). With advancing age, the hippocampus and prefrontal cortex show altered functions. KDs have been tested in its potential to reverse the effects of aging on these two brain regions. KD-fed rats show better performance on a bi-conditional association test, which requires the use of both brain regions (Hernandez et al., 2018). Also in rats, KDs change the gene expression levels in the CA3 region of hippocampus, which is known to impact cognitive abilities during brain aging (Hernandez et al., 2019). These data suggest that KD have the potential to impact the aging brain.

\section{CR and altered intercellular communication}

It has long been noted in the clinical literature that both aging and

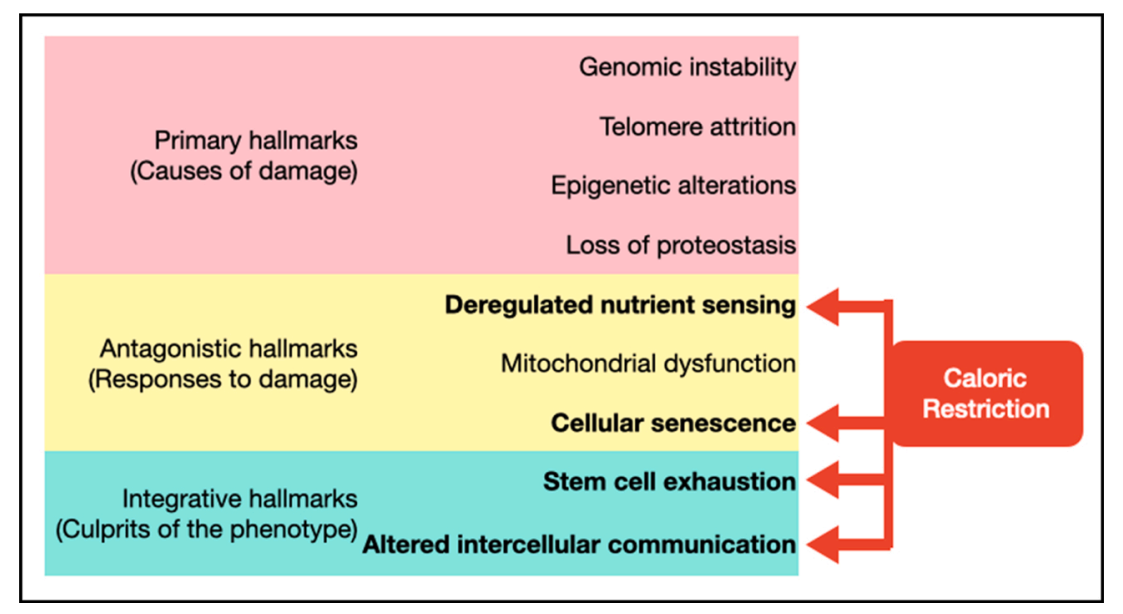

Fig. 1. Caloric restriction and its effects on the hallmarks of aging. 
the nutritional status of the organism, independently, have direct relationships on systemic inflammation, which makes studies related to nutritional interventions critical for alleviating age-related deteriorations (Fig. 2). In terms of the impact of inflammation in the brain, aging was found to be highly associated with microglial activation, accumulation of ROS, increases in the levels of proinflammatory cytokines, and decreases in autophagy (López-Otín et al., 2013). Not surprisingly, it has also been associated with a high prevalence of memory impairments and neuroinflammation-related disease phenotypes such as Alzheimer's, Huntington's, Parkinson's, and prion diseases, as well as in frontotemporal dementia, amyotrophic lateral sclerosis, and chronic traumatic encephalopathy (Guzman-Martinez et al., 2019; Hickman et al., 2018; Si and Liu, 2014; Ward et al., 2015). Microglial sensitization has become a hallmark for the increased neuroinflammatory response in the healthy aging brain and therefore is thought to contribute to the neurodegenerative disease progression (Barrientos et al., 2015). This process occurs even though the primary role of microglia is to protect the brain in response to a specific stimulus, injuries or neuroinflammation. Nevertheless, it also has the potential to harm and kill the neurons when encountering misguided signaling due to altered nutritional status.

With respect to cellular metabolism, ROS elements are produced as byproducts (Chong et al., 2017), whose toxic accumulation increases oxidative stress in the cells with advancing age, and has been associated with endoplasmic reticulum (ER) stress, a state that is induced via accumulation of misfolded proteins, and observed in various diseases (Nishitoh, 2012). Well-known instances for the toxicity resulting from misfolded and unfolded proteins are accumulations of amyloid fibrils and tau proteins in Alzheimer's disease, huntingtin protein in Huntington disease, and $\alpha$ synuclein protein in Parkinson's disease (Martínez et al., 2017; Selkoe, 2003). Creative interventions, which include physical exercise and lowering insulin levels via CR, have been observed to be among some of the efficient approaches to reverse the detrimental processes following increase in ER-stress (Bergamini et al., 2004; Estébanez et al., 2018), and this is in addition to their anti-oxidant and anti-inflammatory properties (Caracciolo et al., 2014; Csiszar et al., 2009; Mohammadi et al., 2014; Santos et al., 2018; Speakman and Mitchell, 2011; Tucsek et al., 2013). One of the mechanisms proposed related to CR-mediated anti-inflammatory effects comes from upregulating nuclear factor-erythroid-2-related factor 2 (Nrf2)-dependent cytoprotective mechanisms that confers protection of the genetic material inside cells as well as integrity of the blood brain barrier (BBB) (Ungvari et al., 2019). Nrf2 regulates the transcription of an array of pro-survival, antioxidant and damage repair genes. Thus its dysregulation by one of the conditions such as aging, obesity or diabetes will in turn induce cellular senescence, which is a cellular response resulting in an inability of cells to continue to divide. As opposed to CR, obesity pathogenesis is associated with harmful side effects on the brain such as hypothalamic inflammation as well as affecting other brain structures like the hippocampus, cortex, brainstem, and amygdala. Gliosis, the brain's injury response, induction of synaptic stripping by microglia, disrupted connectivity, BBB damage, impairment of hippocampal plasticity, and cognitive dysfunction have all been previously associated with obesity and its aging-like symptoms due to systemic inflammation (de Git and Adan, 2015; Dorfman and Thaler, 2015; Guillemot-Legris and Muccioli, 2017; Miller and Spencer, 2014; Fig. 3). One study found that impairment of hippocampal function due to the presence of obesity was correlated with microglial activation, which in turn drives neuroinflammation (Hao et al., 2016). Another study emphasized the anti-aging, health- and lifespan inducing effects of $\mathrm{NAD}^{+}$, a molecule required for mitochondrial ATP production, because declines in cellular $\mathrm{NAD}^{+}$levels are observed with ageing, obesity and diabetes (Csiszar et al., 2019). To counteract obesity-induced pathology, CR has been proposed as an anti-aging strategy that ameliorates or reverses these symptoms by reducing inflammation in the brain alongside of other health benefits such as modifications in oxidative stress, insulin sensitivity, autophagy, and neuroendocrine function (Ellis et al., 2013; Testa et al., 2014).

\section{Deregulated nutrient sensing and CR}

Autophagy, derived from the Latin words for 'self' and 'eating', is the mechanism of lysosome-mediated remodeling of eukaryotic cells by the degradation or recycling of their damaged or dysfunctional components (Tanida, 2011). The role of autophagy in the brain, which delivers toxic molecules to the lysosome, is critical for neuronal health and maintenance. Its involvement in neuroinflammation has drawn scientific interest recently suggesting that impairments in autophagy contribute to the pathogenesis of neurobiological diseases (Cho et al., 2020). According to a large amount of evidence, autophagic system defects in microglia affect inflammation, and immune functions such as phagocytosis in the brain. Autophagic systems must be maintained in balance to provide cellular homeostasis, as too much activation or inhibition would damage the cell. The role of autophagy in processing apoptotic cells, beta amyloids, synaptic materials, myelin wastes, etc. on microglia phagocytosis has been reviewed in the work by Plaza-Zabala et al. (2017). Previous research also found that aging, ER stress, and oxidative stress affect the autophagic function, and alterations in this process are correlated with neurodegeneration found in the aged brain, neurological diseases, and brain injuries (Yin et al., 2017). As a result, increased

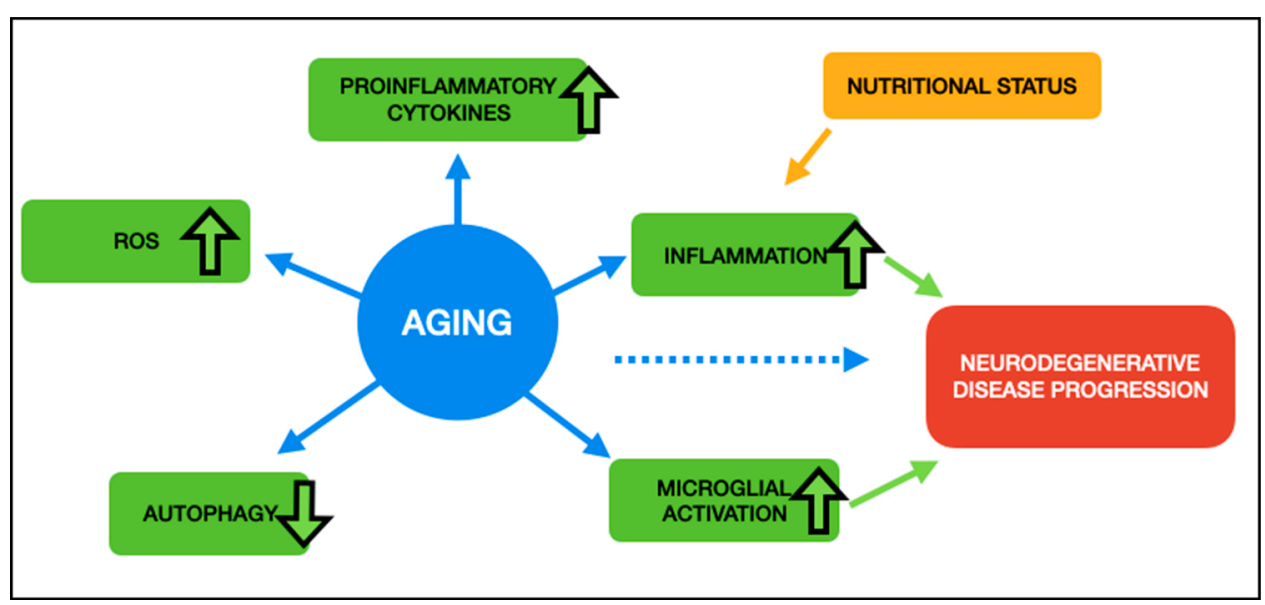

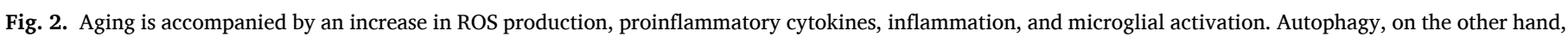

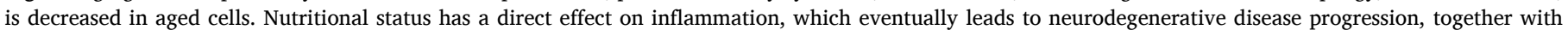
microglial activation and increased cellular aging. 


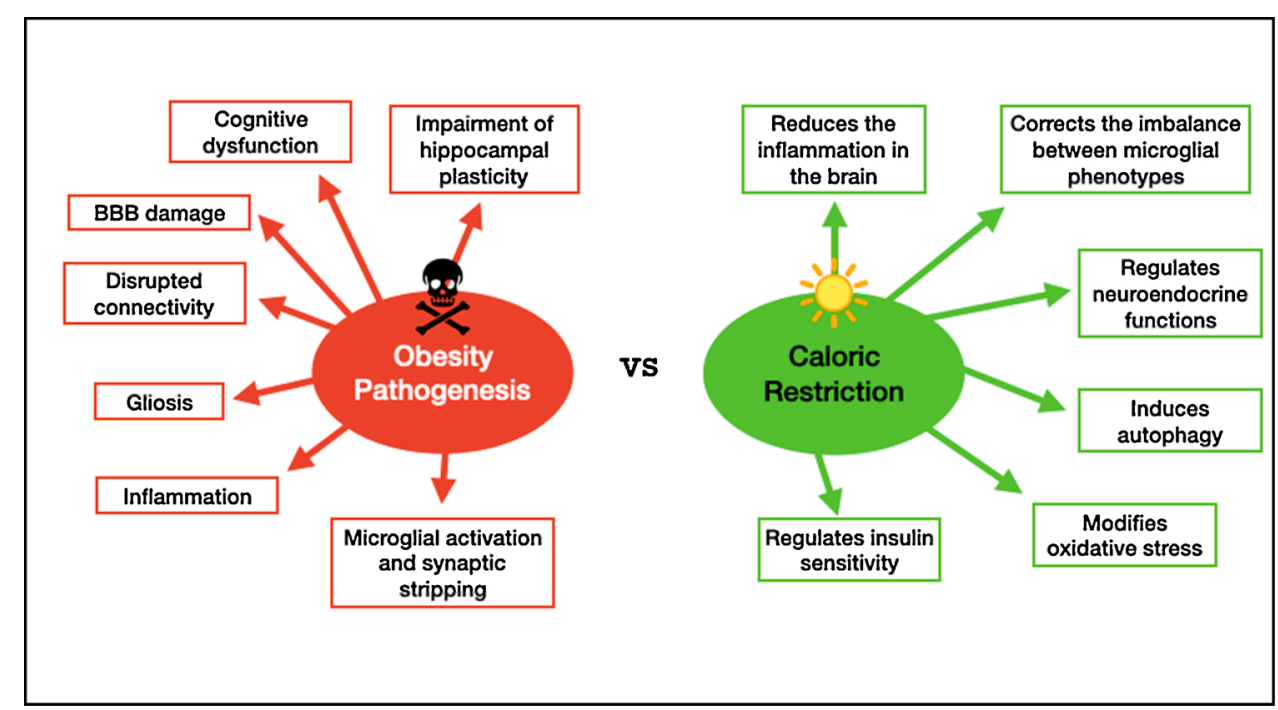

Fig. 3. Caloric restriction has been shown to combat obesity-related changes in inflammation, as an antiaging strategy to reverse the symptoms.

inflammation in the brain is thought to be the leading cause of the pathophysiology behind aging and neurodegenerative diseases. Therefore finding ways for reducing oxidative stress and stimulating autophagy are essential for neuroprotection.

Intermittent fasting or CR has been shown to promote autophagy. The mechanism related to this process and nutrient sensing pathways are believed to be intersecting with the mammalian target of rapamycin (mTOR) pathway, which is thought to be the target of regulation by CR. mTOR is a serine/threonine kinase that is named after its sensitivity to rapamycin, which directly inhibits mTOR activity and its downstream targets (Celebi-Birand et al., 2016). The nutrient deficiency that occurs during fasting or CR also inhibits the mTOR pathway. mTOR complexes are of two distinct protein groups: the target of rapamycin complex 1 (mTORC1) and 2 (mTORC2). CR is known to downregulate mTORC1 (Escobar et al., 2019). mTORC1 is primarily involved in proteome related processes, which also inhibit autophagy through phosphorylation and inhibition of unc 51-like kinase complex (ULK, which is the mammalian equivalent of the autophagy-related protein Atg1; Yin et al., 2017). The ULK complex, ULK1/2 and Atg13, is involved in preautophagosomal formation. Thus, inhibition of mTOR will, in turn, cause stimulation of phagophore formation. Furthermore, as opposed to $\mathrm{CR}$, diabetes has been linked to blockade and defects in autophagy (Muriach et al., 2014). Overall, CR or the mimicking of CR appears to be a desirable and feasible therapeutic intervention to decrease inflammation in the brain by reducing cytokine and ROS levels, promoting autophagy, and correcting the imbalance among microglial phenotypes with a switch from M1 to M2. The cellular mechanisms elongating lifespan through CR seem to be doing this by downregulating cell proliferation, and inducing stem cell quiescence in order to achieve the ultimate aim of saving the stem cell reserve for extreme needs (Cavallucci et al., 2016). Such processes protecting against cellular aging will be reviewed under the cell cycle regulation section.

\section{Stem cell exhaustion}

While cell proliferation is defined as making a copy of one cell after another, in the case of neurons, this does not happen. This is due to the fact that these types of cells become postmitotic after their differentiation. For these cells, the differentiation process is called neurogenesis, which is generating new neurons from neuronal precursors. Neurogenesis continues in a limited fashion into adult life with a decreased rate as compared to earlier developmental periods and being restricted to some discrete areas of the mammalian brain, i.e., subventricular zone (SVZ) of the lateral ventricle, the dentate gyrus of the hippocampus and rostral migratory stream of the olfactory bulb (Eriksson et al., 1998; Apple et al., 2017; Ming and Song, 2011; Taupin, 2006). We also recently showed via RNA sequencing and qPCR validations that stem cell markers were significantly decreasing in the brains of zebrafish with advancing age (Erbaba et al., 2020). Therefore an inverse relationship between aging and stem cell functioning appears to exist.

Alterations in adult neurogenesis have been linked to neurological diseases, brain injury, and stress as well as aging (Apple et al., 2017; Bergmann et al., 2015; Kempermann, 2015; Lee et al., 2000). Previous research studies documented the decline in neuronal generation and turnover rates arising from increased age in rats, mice, and humans (Encinas et al., 2011; Knoth et al., 2010; Kuhn et al., 1996; Lugert et al., 2010; Luo et al., 2006; Maslov et al., 2004; Rao et al., 2006; Sanai et al., 2011). Consequently, decreased progenitor cell proliferation and neuronal differentiation during aging is thought to contribute to reduced plasticity and aging-associated cognitive impairments (Apple et al., 2017).

In order to overcome age-related decreases in overall cell functioning, the literature reviewing neural stem cell (NSC) aging promotes $\mathrm{CR}$ and physical exercise as a protective therapy for healthy brain aging along with improving insulin sensitivity and glucose tolerance, enhancing anti-inflammatory properties, decreasing disease markers, and increasing neuronal plasticity (Apple et al., 2017; Cherif et al., 2016; Kempermann, 2015; Lee et al., 2002; Pani, 2015; Van Praag et al., 1999; Van Praag et al., 2014). The effects of long- and short-term CR have demonstrated the promotion of stem/progenitor cell proliferation in the hippocampus of adult mice and rats, as well as aged animals in several studies (Hornsby et al., 2016; Park et al., 2013). Research shows that there is an increase in the rate of neurogenesis with CR, but the data suggest that these results are due to a decreasing rate of death of newly produced cells rather than an increase in overall cell proliferation (Lee et al., 2000). The mechanisms of how CR exerts its protective effects on the brain and whether there is any direct relationship with neurogenesis is an important question for researchers aimed at discovering potential therapeutic targets. One proposed mechanism to unravel is whether or not the fine tuning of NSCs follows quiescence or proliferation pathways. More specifically, lower nutrient intake or enhanced energy expenditure lead to the quiescence of the NSCs, while elevated calorie intake and anabolic growth signals cause excessive proliferation of these cells. In the case of obesity for example, this excessive proliferation scenario will then eventually result in the depletion of the stem cell reserve, which could be detrimental to the adult brain. This mechanism seems to follow somehow the well-known "Live fast, die young" belief. 


\section{Cellular senescence and CR}

The question of whether or not CR influences cell cycle regulation has been studied. Previous work demonstrated that CR inhibits cell proliferation in the mice thymus, spleen, bone marrow, and kidney tissues (Lu et al., 1993). The negative correlation between CR and cell cycle was also found in a study analyzing human transcriptome datasets on different tissues, and this was probably due to the energetically expensive nature of cell proliferation, which likely requires higher food consumption (Seim et al., 2016). Similarly, another article testing skeletal muscle cells indicated that there is a metabolic shift from proliferation to maintenance and repair, which guides the cells into a younger transcriptional profile (Mercken et al., 2013). It has been hypothesized that the decrease in cell proliferation with CR will increase the time cells spend in the G0/G1 phase of the cell cycle, where the damaged cells will have more time for DNA repair before entering into $S$ phase (Charnley and Tannenbaum, 1985). Conversely, increasing cell proliferation has been associated with decreased time for DNA repair, enhancing the risk for unrepaired DNA synthesis, which eventually increases DNA damage and leads to cancer (Tudek et al., 2010). This outcome of decreased proliferation suggests a unique protective mechanism promoted by CR against cancer as well as possibly aging, by considering the association between DNA damage accumulation due to a low DNA repair capacity that occurs with advanced age (Gensler and Bernstein, 1981; Nicolai et al., 2015; Warner and Price, 1989). Thus it seems that the benefits of CR on life- and healthspan are strongly associated with its function on cell cycle regulation.

One interesting article written by Seim et al. (2016) examines transcriptomes of 21 somatic cell types and tissues, including the brain from publicly available RNA sequencing datasets for the assessment of cell/tissue type-specific turnover rates and cellular lifespans. They provide robust estimates on gene expression level differences between exceptionally long-lived cells with the lowest turnover rates, such as neurons and heart muscle, and the ones having shorter lifespans with highest turnover rates, which include blood and epidermal skin cells. They indicate a statistically significant reduction of the tumor suppression protein $\mathrm{p} 53$ (TP53) in neurons ( $\leq 0.001$ ) as compared to other cell types. Also according to their data, turnover rates were found to be negatively correlated with cell cycle maintenance related factors like CDK regulators such as CCNA2, CCNB2, CHEK1, CHEK2, the proteins which support genomic integrity between cell cycle phases. These statements are consistent with those suggesting a suppressive effect of $\mathrm{CR}$ on cell cycle progression for promoting the cells to spend more time for genomic stability, which in the end decreases the cancer risk. Long-lived neurons having low turnover rates were found to possess a gene expression profile of reduced protein metabolism, which is similar to the expression levels of those associated with CR-mediated lifespan extension (Mayer and Grummt, 2006; Stout et al., 2013). Thus, from this point, the picture dictating the preservation mechanism of neurons that provide them a long lifespan and protection from cancer could be related to lowered amounts of cell cycle-related proteins due to reduced levels of TP53, which in turn inhibits apoptosis for the survival of the differentiated postmitotic cells (Campisi and Di Fagagna, 2007; Ferreira and Kosik, 1996).

Disturbances in cell cycle protein expression are closely tied to neurodegenerative disease progression (Copani et al., 2007; van Leeuwen and Hoozemans, 2015; Ting et al., 2014). The differentiated mature neurons, which normally stay in phase G0 cell cycle arrest, can reenter the cell cycle due to some external or internal factors. This abnormal process results in dedifferentiation and, according to one hypothesis, leads to apoptosis and raises the phosphorylation of tau proteins, which can then promote Alzheimer's Disease progression (Ueberham and Arendt, 2005). Thus, cell cycle regulators are an attractive marker to be investigated further for a relationship to age-related brain pathology and may be selected as markers of healthy versus accelerated brain aging.

\subsection{Transcription factor DP1 (TFDP1) as a possible regulator of brain aging}

In the search for identification of novel molecular signatures of healthy versus flawed aging brain, here we suggest that the gene encoding TFDP1, previously implicated as a factor in cell cycle regulation, may play a key role. TFDP1 is a G1 cell cycle-related protein, whose gene is known to encode a member of a family of transcription factors that join and form a complex with E2F proteins, which are implicated in promoting the $S$ phase in the cell cycle. TFDP1 stimulates the E2Fdependent transcription from a set of target genes by enhancing their binding activity to DNA (Vaishnav and Pant, 1999). The resulting protein complex controls the transcription of many genes that have roles in cell cycle progression from the G1 to $S$ transition. The DP1 transcription factor/E2F complex binds DNA through the E2 recognition site that is found in the promoter region of several genes related to cell cycle regulation and in DNA replication. Cell proliferation and apoptosis are also mediated by the E2F1/DP complex (Chan et al., 2002; NCBI, 2018).

TFDP1-mediated cell cycle regulation could, therefore, be playing a pivotal role in NSC proliferation adjustments via regulation of nutrient uptake. Neurogenic cell fate decisions seem to play an essential role during aging for stem cell reserve maintenance. So the answer to how CR promotes life- and healthspan could be lying underneath this preservation mechanism. However, it needs to be verified via experimental studies that investigate the effects of $\mathrm{CR}$ on cell cycle regulation mechanisms through a TFDP1-mediated transcriptional downregulation.

Although no association has been made neither between TFDP1 and aging, nor TFDP1 and CR effects on the brain, there is an enormous amount of studies pointing to the link between overexpression of TFDP1 and cancer rate incidence. TFDP1 expression was shown to be upregulated in colorectal carcinoma when compared to normal mucosa (Pellatt et al., 2018). In another study, higher expression of stathmins (STMN1), oncoproteins that are critical for the regulation of the microtubule cytoskeleton in nasopharyngeal carcinoma, were correlated with advancing age, and their upregulation with aging is thought to be attributed to E2F1 and TFDP1 transactivation (Hsu et al., 2014). Similarly, Yasui et al. (2002) also mention about the possible role of TFDP1 and its downstream genes on hepatocellular carcinomas in their work. These oncogenic regulations regarding elevated levels of transcription factor DP1 are consistent with a finding suggesting a regulatory mechanism involving p53 and retinoblastoma, tumor suppressor proteins. One study mentions the role of p53, which induces cell cycle arrest by forcing the cell into $\mathrm{G} 1$ entry, in the regulation of transcription driven by the E2F1/DP1 complex. According to their findings, p53 was suggested to compete with E2F1, which induces entry into $S$ phase by DP1 and thereby reducing the DNA binding activity of the DP-1/E2F-1 heterodimer which was shown with a band shift assay measuring DNA binding activity of DP1 (Sørensen et al., 1996; Fig. 4). The data suggests that p53 and E2F1 were cooperating for the homeostatic fine-tuning. If this mechanism is valid, then this would provide a decision switch between proliferation and cell cycle arrest, which puts the role of the TFDP1 protein at the center in controlling the outcome. Another study showed that the retinoblastoma tumor suppressor protein $(\mathrm{Rb})$ uses E2F, the regulator of cell cycle passage, as an inhibitory control mechanism of transcriptional activation (Wu et al., 1995). Other than its function in cancer progression, induced levels of TFDP1 and its partner E2F were also observed in rat hippocampus after global cerebral ischemia together with its dimerization partner, the Rb binding protein, E2F1 (Jin et al., 2001). Lastly, knockdown of TFDP1 was found to reduce PITX1, whose loss of expression has been reported in patients suffering from osteoarthritis, a type of joint inflammation (Pellicelli et al., 2016).

Although thus far no study has provided a direct link between TFDP1 and aging, an indirect correlation between cancer incidence and aging has been made in terms of sharing common mutational patterns (Podolskiy et al., 2016). There is one study which demonstrated that reducing E2F/DP activity leads to senescence-like cell cycle arrest by 


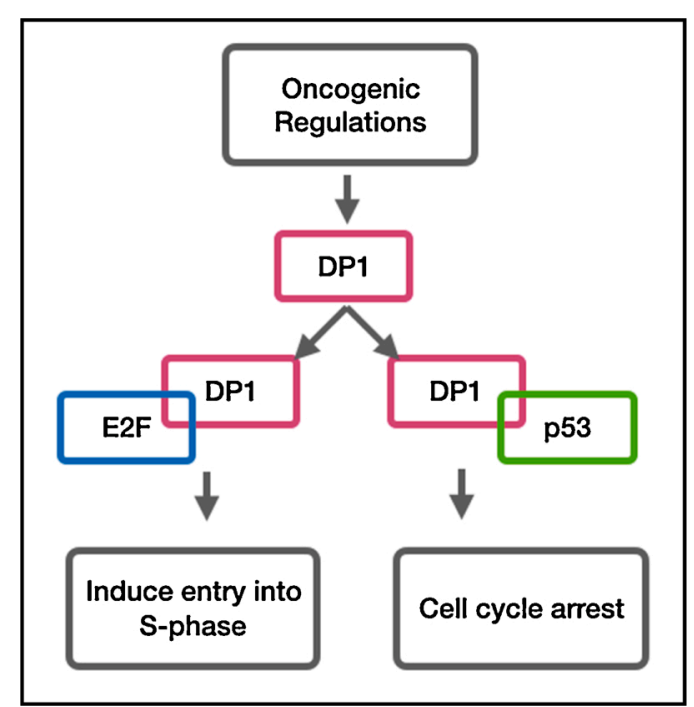

Fig. 4. TFDP1 competes with E2F and p53 depending on the cellular needs, which results in either cell proliferation or cell cycle arrest and senescence.

decreasing the expression of many E2F target genes (Maehara et al., 2005). This is in agreement with the definition of senescence which is a permanent exit from the cell cycle Also there are several other studies investigating senescence with the role of retinoblastoma $(\mathrm{Rb})$ in repressing E2F and a subset of E2F regulated genes involved in DNA replication (Chandler and Peters, 2013; Chicas et al., 2010; Xie et al., 2014). Similarly, Timchenko (2009) reviews the role of E2F in the regeneration of aging livers by stating that silencing the E2F dependent promoters reduces the regenerative capability of livers in aged mice.

Moreover, the search for inferring a relationship between TFDP1 and aging has occurred in several microarray expression datasets that were reviewed by our group. The whole transcriptome was spanned in order to examine TFDP1 behavior against certain factors. A supportive insight has been gathered by reanalysis of such data with the GEO2R tool with respect to the connection among TFDP1 expression with aging and nutritional status of the organism. One such study comes from a GEO dataset (GSE36192; Hernandez et al., 2012), in which 911 frozen post mortem human brain tissues from the cerebellum and frontal cortex regions were collected from 396 human subjects. Since almost all age ranges were covered in the study, the setup allows the user to choose the age range of comparison groups for the analysis. A young male group was chosen from ages that ranged between 13 to 25 years old and an aged male group that was over 80 years old. The resulting GEO2R analysis applied showed significant downregulation of TFDP1 with age in the frontal cortex ( $p=8.63 \mathrm{e}^{09}$; Unpublished data). Another GEO2R analysis applied to a different dataset (GSE26168; Karolina et al., 2011), suggested that TFDP1 significantly upregulates in the blood of type2 diabetic patients as compared to healthy control samples, which supports the hypothesis of a downregulation of this gene with CR ( $p=$ 0.00336; Unpublished data).

While there is no known direct association between Alzheimer's disease and TFDP1 protein regulation in the literature, there is evidence connecting neurodegenerative diseases and the role of TFDP1 in neuronal cell cycle regulation. In one study, Cdk5 is presented as a neuronal protective agent with a functional role in suppressing cell cycle via disruption of the DP1/E2F1 dimerization that regulates cell cycle activation, as well as suppressing neuronal cell death (Liu et al., 2016). Cdk5 coupled with p35 inhibits the dimerization of TFDP1 and E2F1, which gives rise to a pause in the cell cycle (Liu et al., 2016). When the Cdk5 levels decrease, cell death is induced. However, high levels of Cdk5 have been found in the affected regions of Alzheimer's disease brains. Therefore, it has been hypothesized that the excess Cdk5 delays cell death in Alzheimer's disease (Zhang et al., 2010; Zhang and Herrup,
2011). This statement may also provide support for the anticancer and protective effects of TFDP1 downregulation with CR. Reanalysis of a transcriptome dataset with the GEO2R tool for TFDP1 gene expression was performed to assess whether there are any converging pathways of Alzheimer's disease with TFDP1. In the following GEO2R analysis we used a dataset (GSE36980) comprising of 3 different brain regions of both Alzheimer's disease and non- Alzheimer's disease human postmortem tissues (Hokama et al., 2014). According to this analysis, TFDP1 was significantly downregulated in the two brain regions of Alzheimer's disease patients, both the frontal cortex $\left(p=0.00062 ; \mathrm{N}_{\mathrm{AD}}=15, \mathrm{~N}_{\text {non- } \mathrm{AD}}\right.$ $=18)$ and temporal cortex $\left(p=0.015 ; \mathrm{N}_{\mathrm{AD}}=10, \mathrm{~N}_{\text {non- } \mathrm{AD}}=19\right)$, as well as showing there being a marginally significant downregulation in the Alzheimer's disease hippocampi samples ( $p=0.0789 ; \mathrm{N}_{\mathrm{AD}}=7, \mathrm{~N}_{\text {non- } \mathrm{AD}}$ $=10$; Unpublished data). This result raises the question of whether it is possible to induce the adult neural stem cells via TFDP1 promotion to compensate for the neuronal loss due to Alzheimer's disease, which needs to be thoroughly investigated.

\section{Concluding remarks}

This review raises the following question of whether alterations in TFDP1 gene expression and its downstream targets affect the course of aging in the brain, and if so, can we mimic these alterations via dietary strategies and design new therapeutic solutions. If we can find a way to slow down the molecular aging transformations in the brain, then we can also interfere with the increasing rate of the risks of neurodegenerative disease that come with advancing age during the normal lifespan. The main aim of this review is to identify the protective cell cycle and cell proliferation mechanisms surrounding TfDp1-regulated genetic pathways in the brain, by comparing the ones occurring during ad libitum fed natural aging, versus aging following a CR intervention. This investigation of the literature was made to help discover any potential relationships that exist within the nutritional status on the rate of brain aging from the perspective of CR-mediated mechanisms on cell cycle regulations.

To our knowledge, there is no study that directly examines the behavior of TFDP1 neither in the context of CR nor aging in the healthy brain. Also, there is no work investigating the relationship between the role of TFDP1 on NSC proliferation and the brain in the context of CR, which is known to extend lifespan. TFDP1 has been predominantly implicated in cell cycle regulation (Wu et al., 1995), developmental proliferation (Dagnino et al., 1997; Kohn et al., 2003) and cancer progression in various tissues (Tilli et al., 2003; Yasui et al., 2003; Melchor et al., 2009; Chen et al., 2014; Munro et al., 2014; Zhan et al., 2017; Lu et al., 2016; Jibrim et al., 2019). Our review is aimed at merging some of the known effects of CR and cell cycle maintenance on the aging brain. However, more research is needed in making stronger connections between CR and cell cycle regulation, especially with respect to TFDP1, for a better understanding of how they modulate lifespan extension, as well as potentially reverse the neurobiological changes that occur during brain aging and neurodegenerative disease progression.

\section{Funding}

This work was supported by a European Molecular Biology Organization (EMBO) Installation Grant to Michelle Adams provided by The Scientific And Technological Research Council Of Turkey (TUBITAK) and TUBITAK grant number 114 S548.

\section{CRediT authorship contribution statement}

Begun Erbaba: Conceptualization, Methodology, Investigation, Data curation, Writing - original draft. Ayca Arslan-Ergul: Conceptualization, Supervision. Michelle M. Adams: Conceptualization, Supervision, Resources, Writing - review \& editing. 


\section{Declaration of Competing Interest}

The authors declare that they have no competing interests.

\section{References}

Al-Regaiey, K.A., 2016. The effects of calorie restriction on aging: a brief review. Eur. Rev. Med. Pharmacol. Sci. 20 (11), 2468-2473.

Apple, D.M., Solano-Fonseca, R., Kokovay, E., 2017. Neurogenesis in the aging brain. Biochem. Pharmacol. 141, 77-85.

Arslan-Ergul, A., Ozdemir, A.T., Adams, M.M., 2013. Aging, neurogenesis, and caloric restriction in different model organisms. Aging Dis. 4 (4), 221.

Arslan-Ergul, A., Erbaba, B., Karoglu, E.T., Halim, D.O., Adams, M.M., 2016. Short-term dietary restriction in old zebrafish changes cell senescence mechanisms. Neuroscience 334, 64-75.

Barrientos, R.M., Kitt, M.M., Watkins, L.R., Maier, S.F., 2015. Neuroinflammation in the normal aging hippocampus. Neuroscience 309, 84-99.

Bergamini, E., Cavallini, G., Donati, A., Gori, Z., 2004. The role of macroautophagy in the ageing process, anti-ageing intervention and age-associated diseases. Int. J. Biochem. Cell Biol. 36 (12), 2392-2404.

Bergmann, O., Spalding, K.L., Frisén, J., 2015. Adult neurogenesis in humans. Cold Spring Harb. Perspect. Biol. 7 (7), a018994.

Campisi, J., Di Fagagna, F.D.A., 2007. Cellular senescence: when bad things happen to good cells. Nat. Rev. Mol. Cell Biol. 8 (9), 729-740.

Caracciolo, B., Xu, W., Collins, S., Fratiglioni, L., 2014. Cognitive decline, dietary factors and gut-brain interactions. Mech. Ageing Dev. 136, 59-69.

Cavallucci, V., Fidaleo, M., Pani, G., 2016. Neural stem cells and nutrients: poised between quiescence and exhaustion. Trends Endocrinol. Metab. 27 (11), 756-769.

Celebi-Birand, E.D., Karoglu, E.T., Doldur-Balli, F., Adams, M.M., 2016. Mammalian target of rapamycin (mTOR), aging, neuroscience, and their association with aging related diseases. Molecules to Medicine With mTOR. Academic Press, pp. 185-203.

Cerletti, M., Jang, Y.C., Finley, L.W., Haigis, M.C., Wagers, A.J., 2012. Short-term calorie restriction enhances skeletal muscle stem cell function. Cell Stem Cell 10 (5), 515-519.

Chan, J.A., Olvera, M., Lai, R., Naing, W., Rezk, S.A., Brynes, R.K., 2002. Immunohistochemical expression of the transcription factor DP-1 and its heterodimeric partner E2F-1 in non-Hodgkin lymphoma. Appl. Immunohistochem. Mol. Morphol. 10 (4), 322-326.

Chandler, H., Peters, G., 2013. Stressing the cell cycle in senescence and aging. Curr. Opin. Cell Biol. 25 (6), 765-771.

Charnley, G., Tannenbaum, S.R., 1985. Flow cytometric analysis of the effect of sodium chloride on gastric cancer risk in the rat. Cancer Res. 45 (11 Part 2), 5608-5616.

Chen, C., Liu, J., Zhou, F., Sun, J., Li, L., Jin, C., et al., 2014. Next-generation sequencing of colorectal cancers in Chinese: identification of a recurrent frame-shift and gain-offunction indel mutation in the TFDP1 gene. Omics A J. Integr. Biol. 18 (10), 625-635.

Cherif, A., Roelands, B., Meeusen, R., Chamari, K., 2016. Effects of intermittent fasting, caloric restriction, and Ramadan intermittent fasting on cognitive performance at rest and during exercise in adults. Sport. Med. 46 (1), 35-47.

Chicas, A., Wang, X., Zhang, C., McCurrach, M., Zhao, Z., Mert, O., et al., 2010. Dissecting the unique role of the retinoblastoma tumor suppressor during cellular senescence. Cancer Cell 17 (4), 376-387.

Cho, K.S., Lee, J.H., Cho, J., Cha, G.H., Song, G.J., 2020. Autophagy modulators and neuroinflammation. Curr. Med. Chem. 27 (6), 955-982.

Chong, W.C., Shastri, M.D., Eri, R., 2017. Endoplasmic reticulum stress and oxidative stress: a vicious nexus implicated in bowel disease pathophysiology. Int. J. Mol. Sci. 18 (4), 771.

Copani, A., Caraci, F., Hoozemans, J.J., Calafiore, M., Sortino, M.A., Nicoletti, F., 2007. The nature of the cell cycle in neurons: focus on a "non-canonical" pathway of DNA replication causally related to death. Biochimica et Biophysica Acta (BBA)-Mol. Basis Disease 1772 (4), 409-412.

Csiszar, A., Labinskyy, N., Jimenez, R., Pinto, J.T., Ballabh, P., Losonczy, G., et al., 2009. Anti-oxidative and anti-inflammatory vasoprotective effects of caloric restriction in aging: role of circulating factors and SIRT1. Mech. Ageing Dev. 130 (8), 518-527.

Csiszar, A., Tarantini, S., Yabluchanskiy, A., Balasubramanian, P., Kiss, T., Farkas, E., et al., 2019. Role of endothelial NAD + deficiency in age-related vascular dysfunction. Am. J. Physiol. Heart Circul. Physiol. 316 (6), H1253-H1266.

Dagnino, L., Fry, C.J., Bartley, S.M., Farnham, P., Gallie, B.L., Phillips, R.A., 1997. Expression patterns of the E2F family of transcription factors during mouse nervous system development. Mech. Dev. 66 (1-2), 13-25.

de Cabo, R., Mattson, M.P., 2019. Effects of intermittent fasting on health, aging, and disease. N. Engl. J. Med. 381 (26), 2541-2551.

de Diego, I., Peleg, S., Fuchs, B., 2019. The role of lipids in aging-related metabolic changes. Chem. Phys. Lipids 222, 59-69.

de Git, K.C.G., Adan, R.A.H., 2015. Leptin resistance in diet-induced obesity: the role of hypothalamic inflammation. Obes. Rev. 16 (3), 207-224.

Dorfman, M.D., Thaler, J.P., 2015. Hypothalamic inflammation and gliosis in obesity. Curr. Opin. Endocrinol. Diabetes Obes. 22 (5), 325.

Ellis, A., Crowe, K., Lawrence, J., 2013. Obesity-related inflammation: implications for older adults. J. Nutr. Gerontol. Geriatr. 32 (4), 263-290.

Encinas, J.M., Michurina, T.V., Peunova, N., Park, J.H., Tordo, J., Peterson, D.A., et al., 2011. Division-coupled astrocytic differentiation and age-related depletion of neural stem cells in the adult hippocampus. Cell Stem Cell 8 (5), 566-579.
Erbaba, B., Burhan, Ö.P., Şerifoğlu, N., Muratoğlu, B., Kahveci, F., Adams, M.M., ArslanErgül, A., 2020. Zebrafish brain RNA sequencing reveals that cell adhesion molecules are critical in brain aging. Neurobiol. Aging.

Eriksson, P.S., Perfilieva, E., Björk-Eriksson, T., Alborn, A.M., Nordborg, C., Peterson, D. A., Gage, F.H., 1998. Neurogenesis in the adult human hippocampus. Nat. Med. 4 (11), 1313.

Escobar, K.A., Cole, N.H., Mermier, C.M., VanDusseldorp, T.A., 2019. Autophagy and aging: maintaining the proteome through exercise and caloric restriction. Aging Cell 18 (1), e12876.

Estébanez, B., de Paz, J.A., Cuevas, M.J., González-Gallego, J., 2018. Endoplasmic reticulum unfolded protein response, aging and exercise: an update. Front. Physiol. 9, 1744.

Feldman, E.B. (Ed.), 2013. Nutrition in the Middle and Later Years. ButterworthHeinemann.

Ferreira, A., Kosik, K.S., 1996. Accelerated neuronal differentiation induced by p53 suppression. J. Cell. Sci. 109 (6), 1509-1516.

Gensler, H.L., Bernstein, H., 1981. DNA damage as the primary cause of aging. Q. Rev. Biol. 56 (3), 279-303.

Guillemot-Legris, O., Muccioli, G.G., 2017. Obesity-induced neuroinflammation: beyond the hypothalamus. Trends Neurosci. 40 (4), 237-253.

Guzman-Martinez, L., Maccioni, R.B., Andrade, V., Navarrete, L.P., Pastor, M.G., RamosEscobar, N., 2019. Neuroinflammation as a common feature of neurodegenerative disorders. Front. Pharmacol. 10, 1008.

Hao, S., Dey, A., Yu, X., Stranahan, A.M., 2016. Dietary obesity reversibly induces synaptic stripping by microglia and impairs hippocampal plasticity. Brain Behav. Immun. 51, 230-239.

Hernandez, D.G., Nalls, M.A., Moore, M., Chong, S., Dillman, A., Trabzuni, D., et al., 2012. Integration of GWAS SNPs and tissue specific expression profiling reveal discrete eQTLs for human traits in blood and brain. Neurobiol. Dis. 47 (1), 20-28. Dataset: GEO Accession Number: GSE36192 (https://www.ncbi.nlm.nih.gov/geo/ query/acc.. gi? acc $=$ GSE36192).

Hernandez, A.R., Hernandez, C.M., Campos, K., Truckenbrod, L., Federico, Q., Moon, B., et al., 2018. A ketogenic diet improves cognition and has biochemical effects in prefrontal cortex that are dissociable from hippocampus. Front. Aging Neurosci. 10, 391.

Hernandez, A.R., Hernandez, C.M., Truckenbrod, L.M., Campos, K.T., McQuail, J.A., Bizon, J.L., Burke, S.N., 2019. Age and ketogenic diet have dissociable effects on synapse-related gene expression between hippocampal subregions. Front. Aging Neurosci. 11, 239.

Hickman, S., Izzy, S., Sen, P., Morsett, L., El Khoury, J., 2018. Microglia in neurodegeneration. Nat. Neurosci. 21 (10), 1359.

Hokama, M., Oka, S., Leon, J., Ninomiya, T., Honda, H., Sasaki, K., Iwaki, T., Ohara, T., Sasaki, T., LaFerla, F.M., Kiyohara, Y., Nakabeppu, Y., 2014. Altered expression of diabetes-related genes in Alzheimer's disease brains: the Hisayama study. Cereb. Cortex 24 (9), 2476-2488. Dataset: GEO Accession Number: GSE36980 (https ://www.ncbi.nlm.nih.gov/geo/query/acc.cgi?acc=GSE36980).

Hornsby, A.K., Redhead, Y.T., Rees, D.J., Ratcliff, M.S., Reichenbach, A., Wells, T., et al., 2016. Short-term calorie restriction enhances adult hippocampal neurogenesis and remote fear memory in a Ghsr-dependent manner. Psychoneuroendocrinology 63, 198-207.

Hsu, H.P., Li, C.F., Lee, S.W., Wu, W.R., Chen, T.J., Chang, K.Y., et al., 2014. Overexpression of stathmin 1 confers an independent prognostic indicator in nasopharyngeal carcinoma. Tumor Biol. 35 (3), 2619-2629.

Jibrim, R.L.M., de Carvalho, C.V., Invitti, A.L., Schor, E., 2019. Expression of the TFDP1 gene in the endometrium of women with deep infiltrating endometriosis. Gynecol. Endocrinol. 35 (6), 490-493.

Jin, K., Mao, X.O., Eshoo, M.W., Nagayama, T., Minami, M., Simon, R.P., Greenberg, D. A., 2001. Microarray analysis of hippocampal gene expression in global cerebral ischemia. Annals Neurol. Off. J. Am. Neurol. Assoc. Child. Neurol. Soc. 50 (1), 93-103.

Jones, O.R., Scheuerlein, A., Salguero-Gómez, R., Camarda, C.G., Schaible, R., Casper, B. B., et al., 2014. Diversity of ageing across the tree of life. Nature 505 (7482), 169.

Karolina, D.S., Armugam, A., Tavintharan, S., Wong, M.T., Lim, S.C., Sum, C.F., Jeyaseelan, K., 2011. MicroRNA 144 impairs insulin signaling by inhibiting the expression of insulin receptor substrate 1 in type 2 diabetes mellitus. PLoS One 6 (8), e22839. Dataset: GEO Accession Number: GSE26168 (https://www.ncbi.nlm.nih.go $\mathrm{v} /$ geo/query/acc.cgi?acc=GSE26168).

Kempermann, G., 2015. Activity dependency and aging in the regulation of adult neurogenesis. Cold Spring Harb. Perspect. Biol. 7 (11), a018929.

Knoth, R., Singec, I., Ditter, M., Pantazis, G., Capetian, P., Meyer, R.P., et al., 2010. Murine features of neurogenesis in the human hippocampus across the lifespan from 0 to 100 years. PLoS One 5 (1), e8809.

Kohn, M.J., Bronson, R.T., Harlow, E., Dyson, N.J., Yamasaki, L., 2003. Dp1 is required for extra-embryonic development. Development 130 (7), 1295-1305.

Kuhn, H.G., Dickinson-Anson, H., Gage, F.H., 1996. Neurogenesis in the dentate gyrus of the adult rat: age-related decrease of neuronal progenitor proliferation. J. Neurosci. 16 (6), 2027-2033.

Lee, J., Duan, W., Long, J.M., Ingram, D.K., Mattson, M.P., 2000. Dietary restriction increases the number of newly generated neural cells, and induces BDNF expression, in the dentate gyrus of rats. J. Mol. Neurosci. 15 (2), 99-108.

Lee, J., Duan, W., Mattson, M.P., 2002. Evidence that brain-derived neurotrophic factor is required for basal neurogenesis and mediates, in part, the enhancement of neurogenesis by dietary restriction in the hippocampus of adult mice. J. Neurochem. 82 (6), 1367-1375. 
Lettieri-Barbato, D., Cannata, S.M., Casagrande, V., Ciriolo, M.R., Aquilano, K., 2018. Time-controlled fasting prevents aging-like mitochondrial changes induced by persistent dietary fat overload in skeletal muscle. PLoS One 13 (5), e0195912.

Liu, S.L., Wang, C., Jiang, T., Tan, L., Xing, A., Yu, J.T., 2016. The role of Cdk5 in Alzheimer's disease. Mol. Neurobiol. 53 (7), 4328-4342.

Liu, Y., Cheng, A., Li, Y.J., Yang, Y., Kishimoto, Y., Zhang, S., et al., 2019. SIRT3 mediates hippocampal synaptic adaptations to intermittent fasting and ameliorates deficits in APP mutant mice. Nat. Commun. 10 (1), 1-11.

López-Otín, C., Blasco, M.A., Partridge, L., Serrano, M., Kroemer, G., 2013. The hallmarks of aging. Cell 153 (6), 1194-1217.

Lu, M.H., Hinson, W.G., Turturro, A., Sheldon, W.G., Hart, R.W., 1993. Cell proliferation by cell cycle analysis in young and old dietary restricted mice. Mech. Ageing Dev. 68 (1-3), 151-162.

Lu, X., Lv, X.D., Ren, Y.H., Yang, W.D., Li, Z.B., Zhang, L., Bai, X.F., 2016. Dysregulation of TFDP1 and of the cell cycle pathway in high-grade glioblastoma multiforme: a bioinformatic analysis. Genet. Mol. Res. 15 (2).

Lugert, S., Basak, O., Knuckles, P., Haussler, U., Fabel, K., Götz, M., et al., 2010. Quiescent and active hippocampal neural stem cells with distinct morphologies respond selectively to physiological and pathological stimuli and aging. Cell Stem Cell 6 (5), 445-456.

Luo, J., Daniels, S.B., Lennington, J.B., Notti, R.Q., Conover, J.C., 2006. The aging neurogenic subventricular zone. Aging Cell 5 (2), 139-152.

Lyons, L., Schoeler, N.E., Langan, D., Cross, J.H., 2020. Use of ketogenic diet therapy in infants with epilepsy: a systematic review and meta-analysis. Epilepsia.

Maehara, K., Yamakoshi, K., Ohtani, N., Kubo, Y., Takahashi, A., Arase, S., et al., 2005 Reduction of total E2F/DP activity induces senescence-like cell cycle arrest in cance cells lacking functional pRB and p53. J. Cell Biol. 168 (4), 553-560.

Martínez, G., Duran-Aniotz, C., Cabral-Miranda, F., Vivar, J.P., Hetz, C., 2017. Endoplasmic reticulum proteostasis impairment in aging. Aging Cell 16 (4), 615-623.

Maslov, A.Y., Barone, T.A., Plunkett, R.J., Pruitt, S.C., 2004. Neural stem cell detection, characterization, and age-related changes in the subventricular zone of mice. J. Neurosci. 24 (7), 1726-1733.

Mattson, M.P., 2005. Energy intake, meal frequency, and health: a neurobiological perspective. Annu. Rev. Nutr. 25, 237-260.

Mattson, M.P., Longo, V.D., Harvie, M., 2017. Impact of intermittent fasting on health and disease processes. Ageing Res. Rev. 39, 46-58.

Mayer, C., Grummt, I., 2006. Ribosome biogenesis and cell growth: mTOR coordinates transcription by all three classes of nuclear RNA polymerases. Oncogene 25 (48), 6384-6391.

Melchor, L., Saucedo-Cuevas, L.P., Muñoz-Repeto, I., Rodríguez-Pinilla, S.M., Honrado, E., Campoverde, A., et al., 2009. Comprehensive characterization of the DNA amplification at 13q34 in human breast cancer reveals TFDP1 and CUL4A as likely candidate target genes. Breast Cancer Res. 11 (6), R86.

Mercken, E.M., Crosby, S.D., Lamming, D.W., JeBailey, L., Krzysik-Walker, S., Villareal, D.T., et al., 2013. Calorie restriction in humans inhibits the PI 3 K/AKT pathway and induces a younger transcription profile. Aging Cell 12 (4), 645-651.

Meynet, O., Ricci, J.E., 2014. Caloric restriction and cancer: molecular mechanisms and clinical implications. Trends Mol. Med. 20 (8), 419-427.

Miller, A.A., Spencer, S.J., 2014. Obesity and neuroinflammation: a pathway to cognitive impairment. Brain Behav. Immun. 42, 10-21.

Ming, G.L., Song, H., 2011. Adult neurogenesis in the mammalian brain: significant answers and significant questions. Neuron 70 (4), 687-702.

Mohammadi, M., Ghaznavi, R., Keyhanmanesh, R., Sadeghipour, H.R., Naderi, R., Mohammadi, H., 2014. Caloric restriction prevents lead-induced oxidative stress and inflammation in rat liver. Sci. World J. 2014.

Munro, S., Oppermann, U., La Thangue, N.B., 2014. Pleiotropic effect of somatic mutations in the E2F subunit DP-1 gene in human cancer. Oncogene 33 (27), 3594-3603.

Muriach, M., Flores-Bellver, M., Romero, F.J., Barcia, J.M., 2014. Diabetes and the brain: oxidative stress, inflammation, and autophagy. Oxid. Med. Cell. Longev. 2014

NCBI, 2018. CCNB2 Cyclin B2 [Homo sapiens (human)], 2 Oct Retrieved from. https://www.ncbi.nlm.nih.gov/gene/9133.

Nicolai, S., Rossi, A., Di Daniele, N., Melino, G., Annicchiarico-Petruzzelli, M., Raschellà, G., 2015. DNA repair and aging: the impact of the p53 family. Aging (Albany NY) 7 (12), 1050.

Nishitoh, H., 2012. CHOP is a multifunctional transcription factor in the ER stress response. J. Biochem. 151 (3), 217-219.

Pani, G., 2015. Neuroprotective effects of dietary restriction: evidence and mechanisms. April. In: Seminars in Cell \& Developmental Biology, Vol. 40. Academic Press, pp. 106-114.

Park, J.H., Glass, Z., Sayed, K., Michurina, T.V., Lazutkin, A., Mineyeva, O., et al., 2013. Calorie restriction alleviates the age-related decrease in neural progenitor cell division in the aging brain. Eur. J. Neurosci. 37 (12), 1987-1993.

Pellatt, A.J., Mullany, L.E., Herrick, J.S., Sakoda, L.C., Wolff, R.K., Samowitz, W.S., Slattery, M.L., 2018. The TGFß-signaling pathway and colorectal cancer: associations between dysregulated genes and miRNAs. J. Transl. Med. 16 (1), 191.

Pellicelli, M., Picard, C., Wang, D., Lavigne, P., Moreau, A., 2016. E2F1 and TFDP1 regulate PITX1 expression in normal and osteoarthritic articular chondrocytes. PLoS One 11 (11), e0165951.

Plaza-Zabala, A., Sierra-Torre, V., Sierra, A., 2017. Autophagy and microglia: novel partners in neurodegeneration and aging. Int. J. Mol. Sci. 18 (3), 598.

Podolskiy, D.I., Lobanov, A.V., Kryukov, G.V., Gladyshev, V.N., 2016. Analysis of cancer genomes reveals basic features of human aging and its role in cancer development. Nat. Commun. 7, 12157.
Rao, M.S., Hattiangady, B., Shetty, A.K., 2006. The window and mechanisms of major age-related decline in the production of new neurons within the dentate gyrus of the hippocampus. Aging Cell 5 (6), 545-558.

Rusek, M., Pluta, R., Ułamek-Kozioł, M., Czuczwar, S.J., 2019. Ketogenic diet in Alzheimer's disease. Int. J. Mol. Sci. 20 (16), 3892.

Sanai, N., Nguyen, T., Ihrie, R.A., Mirzadeh, Z., Tsai, H.H., Wong, M., et al., 2011. Corridors of migrating neurons in the human brain and their decline during infancy. Nature 478 (7369), 382.

Santos, C.L., Bobermin, L.D., Souza, D.O., Quincozes-Santos, A., 2018. Leptin stimulates the release of pro-inflammatory cytokines in hypothalamic astrocyte cultures from adult and aged rats. Metab. Brain Dis. 1-5.

Seim, I., Ma, S., Gladyshev, V.N., 2016. Gene expression signatures of human cell and tissue longevity. NPJ Aging Mech. Dis. 2 (1), 1-8.

Selkoe, D.J., 2003. Folding proteins in fatal ways. Nature 426 (6968), 900.

Si, H., Liu, D., 2014. Dietary antiaging phytochemicals and mechanisms associated with prolonged survival. J. Nutr. Biochem. 25 (6), 581-591.

Sørensen, T.S., Girling, R., Lee, C.W., Gannon, J., Bandara, L.R., La Thangue, N.B., 1996. Functional interaction between DP-1 and p53. Mol. Cell. Biol. 16 (10), 5888-5895.

Somel, M., Khaitovich, P., Bahn, S., Pääbo, S., Lachmann, M., 2006. Gene expression becomes heterogeneous with age. Curr. Biol. 16 (10), R359-R360.

Speakman, J.R., Mitchell, S.E., 2011. Caloric restriction. Mol. Aspects Med. 32 (3), $159-221$.

Stout, G.J., Stigter, E.C., Essers, P.B., Mulder, K.W., Kolkman, A., Snijders, D.S., et al., 2013. Insulin/IGF-1-mediated longevity is marked by reduced protein metabolism. Mol. Syst. Biol. 9 (1).

Tanida, I., 2011. Autophagy basics. Microbiol. Immunol. 55 (1), 1-11.

Taupin, P., 2006. Adult neural stem cells, neurogenic niches, and cellular therapy. Stem Cell Rev. 2 (3), 213-219.

Taylor, M.K., Swerdlow, R.H., Sullivan, D.K., 2019. Dietary neuroketotherapeutics for Alzheimer's disease: an evidence update and the potential role for diet quality. Nutrients 11 (8), 1910.

Testa, G., Biasi, F., Poli, G., Chiarpotto, E., 2014. Calorie restriction and dietary restriction mimetics: a strategy for improving healthy aging and longevity. Curr. Pharm. Des. 20 (18), 2950-2977.

Tilli, M.T., Hudgins, S.L., Frech, M.S., Halama, E.D., Renou, J.P., Furth, P.A., 2003. Loss of protein phosphatase 2A expression correlates with phosphorylation of DP-1 and reversal of dysplasia through differentiation in a conditional mouse model of cancer progression. Cancer Res. 63 (22), 7668-7673.

Timchenko, N.A., 2009. Aging and liver regeneration. Trends Endocrinol. Metab. 20 (4), $171-176$.

Ting, J.H., Marks, D.R., Schleidt, S.S., Wu, J.N., Zyskind, J.W., Lindl, K.A., et al., 2014. Targeted gene mutation of E2F1 evokes age-dependent synaptic disruption and behavioral deficits. J. Neurochem. 129 (5), 850-863.

Tucsek, Z., Toth, P., Sosnowska, D., Gautam, T., Mitschelen, M., Koller, A., et al., 2013. Obesity in aging exacerbates blood-brain barrier disruption, neuroinflammation, and oxidative stress in the mouse hippocampus: effects on expression of genes involved in beta-amyloid generation and Alzheimer's disease. J. Gerontol. Series A: Biomed. Sci. Med. Sci. 69 (10), 1212-1226.

Tudek, B., Winczura, A., Janik, J., Siomek, A., Foksinski, M., Oliński, R., 2010. Involvement of oxidatively damaged DNA and repair in cancer development and aging. Am. J. Transl. Res. 2 (3), 254.

Ueberham, U., Arendt, T., 2005. The expression of cell cycle proteins in neurons and its relevance for Alzheimer's disease. Curr. Drug Targets-CNS \& Neurol. Disorders 4 (3), 293-306.

Ungvari, Z., Tarantini, S., Nyúl-Tóth, Á., Kiss, T., Yabluchanskiy, A., Csipo, T., et al., 2019. Nrf2 dysfunction and impaired cellular resilience to oxidative stressors in the aged vasculature: from increased cellular senescence to the pathogenesis of agerelated vascular diseases. GeroScience 1-12.

Vaishnav, Y.N., Pant, V., 1999. Differential regulation of E2F transcription factors by p53 tumor suppressor protein. DNA Cell Biol. 18 (12), 911-922.

van Leeuwen, L.A., Hoozemans, J.J., 2015. Physiological and pathophysiological functions of cell cycle proteins in post-mitotic neurons: implications for Alzheimer's disease. Acta Neuropathol. 129 (4), 511-525.

Van Praag, H., Kempermann, G., Gage, F.H., 1999. Running increases cell proliferation and neurogenesis in the adult mouse dentate gyrus. Nat. Neurosci. 2 (3), 266.

Van Praag, H., Fleshner, M., Schwartz, M.W., Mattson, M.P., 2014. Exercise, energy intake, glucose homeostasis, and the brain. J. Neurosci. 34 (46), 15139-15149.

Ward, R.J., Dexter, D.T., Crichton, R.R., 2015. Ageing, neuroinflammation and neurodegeneration. Front. Biosci. Schol. Ed. (Schol Ed) 7, 189-204.

Warner, H.R., Price, A.R., 1989. Involvement of DNA repair in cancer and aging. J. Gerontol. 44 (6), 45-54.

Weithoff, G., 2007. Dietary restriction in two rotifer species: the effect of the length of food deprivation on life span and reproduction. Oecologia 153 (2), 303-308.

Wu, C.L., Zukerberg, L.R., Ngwu, C., Harlow, E., Lees, J.A., 1995. In vivo association of E2F and DP family proteins. Mol. Cell. Biol. 15 (5), 2536-2546.

Xie, Q., Peng, S., Tao, L., Ruan, H., Yang, Y., Li, T.M., et al., 2014. E2F transcription factor 1 regulates cellular and organismal senescence by inhibiting Forkhead box O transcription factors. J. Biol. Chem. 289 (49), 34205-34213.

Yasui, K., Arii, S., Zhao, C., Imoto, I., Ueda, M., Nagai, H., et al., 2002. TFDP1, CUL4A, and CDC16 identified as targets for amplification at 13q34 in hepatocellular carcinomas. Hepatology 35 (6), 1476-1484.

Yasui, K., Okamoto, H., Arii, S., Inazawa, J., 2003. Association of over-expressed TFDP1 with progression of hepatocellular carcinomas. J. Hum. Genet. 48 (12), 609-613.

Yin, Y., Sun, G., Li, E., Kiselyov, K., Sun, D., 2017. ER stress and impaired autophagy flux in neuronal degeneration and brain injury. Ageing Res. Rev. 34, 3-14. 
Zhan, W., Wang, W., Han, T., Xie, C., Zhang, T., Gan, M., Wang, J.B., 2017. COMMD9 promotes TFDP1/E2F1 transcriptional activity via interaction with TFDP1 in nonsmall cell lung cancer. Cell. Signal. 30, 59-66.

Zhang, J., Herrup, K., 2011. Nucleocytoplasmic Cdk5 is involved in neuronal cell cycle and death in post-mitotic neurons. Cell Cycle 10 (8), 1208-1214.
Zhang, J., Li, H., Yabut, O., Fitzpatrick, H., D’Arcangelo, G., Herrup, K., 2010. Cdk5 suppresses the neuronal cell cycle by disrupting the E2F1-DP1 complex. J. Neurosci. 30 (15), 5219-5228. 